\title{
Respiratory Syncytial Virus Infection in Infants with Heart and Lung Diseases
}

\author{
Hiroyuki Yamagishi
}

\begin{abstract}
Respiratory syncytial virus (RSV) is a major causative agent of respiratory infection in infants because of its affinity for bronchial and alveolar epithelial cells. Serious and sometimes fatal RSV infection may develop in premature infants as well as infants with chronic lung disease and congenital heart disease (CHD). In infants with CHD, the addition of RSV infection to the underlying respiratory pathology resulting from increase or decrease in pulmonary blood flow may cause significant problems such as serious symptoms, increases in hospitalization rate, postponement of elective heart surgery, or death. Strict infection control measures with administration of anti-RSV antibody are essential in the management of infants with heart and lung diseases.
\end{abstract}

\subsection{Clinical Characteristics of RSV Infection}

Respiratory syncytial virus (RSV) accounts for more than $30 \%$ of hospitalizations of children under 3 years of age due to respiratory infection [1]. RSV infection typically develops acute upper respiratory tract inflammation associated with watery nasal discharge, cough, and fever. Although RSV infection causes only mild or moderate upper respiratory tract inflammation in most adults and older children, serious RSV infection may develop in babies and young infants who often experience acute bronchiolitis and pneumonia associated with expiratory wheezing and tachypnea. Serious and sometimes fatal RSV infection may develop in infants with

\footnotetext{
H. Yamagishi $(\square)$

Division of Pediatric Cardiology, Department of Pediatrics,

Keio University School of Medicine, Tokyo, Japan

e-mail: hyamag@keio.jp
} 
underlying conditions such as a history of premature birth, chronic lung disease (CLD), and congenital heart disease (CHD).

\subsection{Impact of RSV Infection on CHD}

RSV infection in infants with CHD is of particular significance in the following respects:

1. Severe RSV infection may develop in infants with CHD [2, 3].

(a) The rate of hospitalization due to RSV infection is higher in infants with CHD than in those without CHD.

(b) The use of ICU care and ventilation for the treatment of RSV infection is more common in infants with CHD than in those without CHD.

(c) Mortality due to RSV infection is higher in infants with CHD than in those without CHD.

2. The prognosis of surgical treatment for CHD (open heart surgery) is poor when surgery is performed before complete recovery from RSV infection [4].

\subsection{Respiratory Pathophysiology and Severe RSV Infection in Infants with CHD}

CHD is classified into two main classes: left-to-right shunt lesion associated with congestive heart failure and right-to-left shunt lesion associated with cyanosis. In both types of CHD, infants typically have the respiratory pathophysiological changes which may predispose to severe RSV infection [5].

Normal respiratory function in normal infants features sufficient tidal volume, pulmonary compliance, and airway resistance. It is known that RSV infection decreases alveolar liquid clearance and pulmonary compliance by injuring alveolar epithelial cells and inducing inflammatory edema of tissues surrounding the peripheral pulmonary arteries. Bronchiolitis due to RSV infection causes airway obstruction with increase in airway resistance as well. These changes result in decrease in tidal volume and increase in imbalance of ventilation and blood flow (V/Q mismatch), which promote hypoxemia and hypercapnia.

In cases of CHD with a left-to-right shunt such as ventricular septal defect and patent ductus arteriosus, increases in pulmonary capillary wedge pressure and left atrial pressure due to the increase in pulmonary blood flow are observed, and pulmonary hypertension may occur in some cases. Due to the presence of extravasation of fluid resulting from increased blood flow and pressure in the pulmonary vessels, the excessive fluid leaking into interstitial tissues tends to flow into the alveoli. In cases of significant pulmonary congestion, decrease in pulmonary compliance and increase in airway resistance due to compression of the airways by surrounding dilated vessels may occur, resulting in increase in respiratory rate. When children with such underlying conditions contract RSV infection, alveolar liquid compliance 
and lung compliance will be further decreased, and airway resistance will rapidly increase. As a result, lung tissue swelling and pulmonary edema may develop with decrease in functional residual capacity and rapid deterioration of V/Q mismatch, and severe respiratory signs/symptoms may occur. RSV infection may become particularly serious in children with pulmonary hypertension, who must thus be monitored carefully.

On the other hand, in cases of CHD with right-to-left shunt and decrease in pulmonary blood flow such as the tetralogy of Fallot, cyanosis and hypoxemia are constantly present. Due to the small lung volume and underdevelopment of airways resulting from low pulmonary blood flow, peripheral airway resistance is high and lung compliance is low in such patients. Although this type of CHD is not frequently associated with pulmonary edema, unlike CHD with increased pulmonary blood flow, RSV infection accelerates the increase in airway resistance and decrease in pulmonary compliance with significant deterioration of cyanosis and hypoxemia and development of severe respiratory signs and symptoms.

In cases of complex heart disease such as complete transposition of the great arteries and hypoplastic left heart syndrome, which are characterized by increase in pulmonary blood flow and hypoxemia, mixtures of the above-described pathophysiological conditions are present, and RSV infection induces clinical deterioration.

\subsection{Prevention of RSV Infection in Infants with CHD}

To date, no specific treatment for RSV infection has been established. Therefore, prevention of RSV infection is essential in the management of infants with CHD.

In our hospital, infants and young children $\leq 3$ years of age are usually hospitalized in the infant ward (a large room) with full nursing service. In the infant ward, standard infection control procedures including hand washing when entering and leaving the ward, hand disinfection before and after examining each patient, wearing a gown specific to each patient, and use of a stethoscope specific to each patient are strictly adhered to. Since many children hospitalized in the infant ward have CHD and/or CLD and are at high risk for severe RSV infection, all children to be hospitalized in the infant ward are subjected to a rapid RSV antigen test in the ambulatory setting on the day of hospitalization during RSV infection is prevalent. Children with a positive test for RSV antigen are not allowed to enter the infant ward and are hospitalized in a private room or referred to other hospitals. The first step to preventing RSV infection in infants with CHD is preventing sources of infection from entering the infant ward.

When signs/symptoms of respiratory infection develop in patients hospitalized in the infant ward especially during the period of prevalent RSV infection, they are subjected to a rapid RSV antigen test to detect RSV infection as early as possible. Children with mild RSV infection (a positive RSV antigen test) who do not require ventilation or intensive care are transferred to private rooms with prior consent while children with severe RSV infection requiring ventilation and/or intensive care, who are difficult to care for in private rooms, enter the infant ICU with the 
following measures taken to avoid transmission of RSV infection. Each infected child's bed is surrounded by portable partitions with metal frames and transparent plastic panels to separate the child from others and avoid droplet infection in the crowded infant ward, which features small distances between beds. In addition, a hand washer and a specific door to the infant ward are used only by healthcare professionals and visitors who care for children with RSV infection to ensure separation of them in the infant ward. Children with RSV infection are attended only by designated nurses who do not attend other children.

Education of staff members is essential to ensuring appropriate infection control measures. In the CDC's guidelines, education of hospital staff is rated as Category IA [6]. In our hospital, an infection control section regularly provides "pediatric infection control seminars" by pediatric infection specialists in order to increase awareness of infection control.

\subsection{The Use of Palivizumab in Infants with CHD}

A humanized monoclonal antibody specific to RSV (palivizumab, Synagis ${ }^{\circledR}$ ) was developed and released. Palivizumab is an antibody specific to the antigenic region of the F protein of RSV, neutralizes the infectivity of RSV, inhibits amplification and growth of RSV, and thereby prevents the onset of severe lower respiratory tract disease.

From 1998 to 2002, a multicenter clinical study of palivizumab in CHD patients was conducted in Europe and the United States. A total of 1, 287 children were randomized and allocated to a palivizumab group (639 children) or placebo group (648 children). The rate of hospitalization due to RSV infection was decreased by $45 \%$ in the palivizumab group $(9.7 \%$ in the placebo group vs. $5.3 \%$ in the palivizumab group; $p=0.03$ ) [7]. Palivizumab was administered intramuscularly at a dose of $15 \mathrm{mg} / \mathrm{kg}$ body weight every month throughout the RSV season.

In October 2005, palivizumab was approved for infants with CHD by the National Health Insurance in Japan, and Japanese guidelines for the use of palivizumab in infants with CHD was prepared [8]. Since palivizumab does not affect the efficacy of vaccinations, treatment with it can be continued without changing vaccination schedules. Even if RSV infection develops during treatment with palivizumab, palivizumab should continue to be administered thereafter during the RSV season since reinfection with RSV causing serious disease may occur during the same season.

\subsection{Conclusions and Future Perspectives}

Cox et al. noted that "conventional infection control measures failed to prevent spread of RSV infection, and palivizumab could prevent occurrence of nosocomial RSV infections" [9]. We believe that performance of standard infection control measures considering the mode of transmission of RSV and education of hospital 
staff are important steps in preventing RSV infection in children with CHD. Efforts to detect sources of infection as early as possible and use of preventive treatment with palivizumab are also essential.

It has been generally accepted that palivizumab should be administered from September-October to March-April in Japan. However, a child with a positive RSV antigen test is recently often observed in May, July, and August. Duration of palivizumab treatment should thus be modified based on the prevalence of RSV infection in the relevant geographical area and trends in the incidence of RSV infection in each year. It is preferable that serum palivizumab concentration be high enough to ensure prevention of RSV infection. Physicians should also consider whether to perform additional administration of palivizumab after surgery using cardiopulmonary bypass, which is known to decrease serum palivizumab concentration. Although no serious adverse events related to treatment with palivizumab have been reported, monitoring should be continued to ensure the safety of palivizumab treatment in each year.

\section{References}

1. Kaneko M, Watanabe J, Kuwahara M, et al. Impact of respiratory syncytial virus infection as a cause of lower respiratory tract infection in children younger than 3 years of age in Japan. J Infect. 2002;44:240-3.

2. Boyce TG, Mellen BG, Mitchel EF Jr, et al. Rates of hospitalization for respiratory syncytial virus infection among children in Medicaid. J Pediatr. 2000;137:865-70.

3. Navas L, Wang E, de Carvalho V, Robinson J. Pediatric Investigators Collaborative Network on Infections in Canada; Improved outcome of respiratory syncytial virus infection in a high-risk hospitalized population of Canadian children. J Pediatr. 1992;121:348-54.

4. Khongphatthanayothin A, Wong PC, Samara Y, et al. Impact of respiratory syncytial virus infection on surgery for congenital heart disease: postoperative course and outcome. Crit Care Med. 1999;27:1974-81.

5. MacDonald NE, et al. Respiratory syncytial viral infection in infants with congenital heart disease. N Engl J Med. 1982;307:397-400.

6. Greenough A. Respiratory syncytial virus infection; clinical features, management, and prophylaxis. Curr Opin Pulm Med. 2002;8:214-7.

7. Feltes TF, Cabalka AK, Meissner HC, et al. Palivizumab prophylaxis reduces hospitalization due to respiratory syncytial virus in young children with hemodynamically significant congenital heart disease. J Pediatr. 2003;143:532-40.

8. Nakazawa M, Saji T, Ichida F, et al. Guideline for the use of palivizumab in infants and young children with congenital heart disease. Pediatr Int. 2006;48:190-3.

9. Cox RA, Rao P, Brandon-Cox C, et al. The use of palivizumab monoclonal antibody to control an outbreak of nosocomial respiratory syncytial virus infection in a special care baby unit. J Hosp Infect. 2001;48:186-92. 
Open Access This chapter is licensed under the terms of the Creative Commons Attribution 4.0 International License (http://creativecommons.org/licenses/by/4.0/), which permits use, sharing, adaptation, distribution and reproduction in any medium or format, as long as you give appropriate credit to the original author(s) and the source, provide a link to the Creative Commons license and indicate if changes were made.

The images or other third party material in this chapter are included in the chapter's Creative Commons license, unless indicated otherwise in a credit line to the material. If material is not included in the chapter's Creative Commons license and your intended use is not permitted by statutory regulation or exceeds the permitted use, you will need to obtain permission directly from the copyright holder. 